\title{
Multiionic and Permittivity-Related Effects on the Diffuse Electric Double Layer Structure at Solid-Electrolyte Solution Interfaces
}

\author{
J. J. López-García $\mathbb{D}^{1},{ }^{1}$ J. Horno ${ }^{1},{ }^{1}$ and C. Grosse ${ }^{2}$ \\ ${ }^{1}$ Departamento de Física, Universidad de Jaén, Campus Las Lagunillas, Ed. A-3, 23071 Jaén, Spain \\ ${ }^{2}$ Departamento de Física, Universidad Nacional de Tucumán, Av. Independencia 1800, 4000 San Miguel de Tucumán, Argentina
}

Correspondence should be addressed to J. Horno; jhorno@ujaen.es

Received 8 March 2018; Accepted 27 August 2018; Published 30 September 2018

Academic Editor: Candido Fabrizio Pirri

Copyright (c) 2018 J. J. López-García et al. This is an open access article distributed under the Creative Commons Attribution License, which permits unrestricted use, distribution, and reproduction in any medium, provided the original work is properly cited.

\begin{abstract}
The structure and differential capacitance of the diffuse part of the electric double layer at solid-electrolyte solution interfaces are examined using a theoretical model that takes into account the finite ion size by modeling the solution as a suspension of polarizable insulating spheres in water. This formalism is applied to binary and mixed electrolyte solutions using the "Boublik-Mansoori-Carnahan-Starling-Leland" (BMCSL) theory for the steric interactions among ions. It is shown that the ionic size differences have a strong bearing on the diffuse part of the electric double layer structure, as well as on the differential capacitance dependence on the surface potential for mixed electrolytes.
\end{abstract}

\section{Introduction}

It is well-known that when solid objects are placed in contact with an aqueous electrolyte solution, they usually acquire a surface charge and spatial distributions of charge and electric potential, known as the electric double layer (EDL), appear close to the interfaces. This structure plays a crucial role in materials science, electrochemistry, colloid and polymer science, biophysics, and the study of nanofluidic devices [1-3].

Helmholtz [4] was the first to show that an EDL is essentially a molecular condenser able to store charge. This early model predicted a constant differential capacitance value dependent on the permittivity of the electrolyte solution $\varepsilon_{e}$ and the thickness of the double layer $d$ but independent of the surface charge density.

Later Gouy and Chapman [5, 6] made significant improvements by considering that the charge distribution of ions as a function of distance from the solid-liquid interface follows the Maxwell-Boltzmann statistics. However, important simplifications were also assumed: ions are treated as mathematical points; non-Coulombic interactions between counterions, co-ions, and the solid are disregarded; the macroscopic permittivity value $\varepsilon_{e}$ is considered to be constant in the whole ionic solution up to the very surface of the solid; incomplete dissociation of the electrolyte is ignored; and so on.

In 1924, Stern [7] considered that ions, because of their finite volume, cannot come infinitely close to the solid surface. He so suggested a combination of the Helmholtz and the Gouy-Chapman models. In Stern's model, ions in contact with the solid surface behave as in the Helmholtz model, while the remaining ions, located outside this Stern layer, form a diffuse Gouy-Chapman layer.

Most of the EDL interpretations that followed continued using the original Stern idea, i.e., to divide the EDL into two parts: an inner part in contact with the solid surface and a diffuse electric double layer (DEDL). In most of these studies, it was further assumed that the remaining discrepancies with experimental results originated from an overly simplified model of the inner layer, which has been corrected including specific ionic adsorption, solvent reduction, strong metal-solvent interactions, etc. [8-12]. However, in the last decades, several authors suggested that differences between theoretical model predictions and experimental data may be due to the assumption of ions as 
mathematical points in the Gouy-Chapman model for the DEDL.

Roughly, two types of methods have been used to include ion size effects: microscopic descriptions of the system with different approximation levels and phenomenological theories using macroscopic differential equations to describe the average behavior of the system. Microscopic descriptions have the advantage of a precise representation of the interactions responsible for the macroscopic behavior but only in equilibrium (out of equilibrium studies are possible in principle but at an enormous computational cost). On the contrary, phenomenological theories, less strict in the description of the interactions, make it possible in practice to analyze the system behavior both in equilibrium and perturbed by an external electric field (see reference [13] and references cited within).

Basically, all the phenomenological models consider that ions have an effective ionic radius so that their local concentration cannot surpass a finite value and introduce an activity coefficient in order to account for both the ionic size and the steric interactions among ions. While different expressions have been used for the activity coefficient, most of the studies published to date use the equation proposed by Bikerman in 1942 [14]. This expression only takes into account the excluded ionic volume neglecting all other steric effects and solely depends on the local volume fraction occupied by the ions so that it has the same value for all the ionic species present in the solution. The results of this modification of the standard model have shown that steric interactions are crucial for the interpretation of the equilibrium double layer of electrodes when both the surface charge and the bulk ionic concentration are high. For colloidal suspensions, however, where these parameters have typically much lower values, finite ion size effects appeared at first to be almost negligible [15].

In recent years, the theoretical model was extended considering three main aspects. The first consists in realizing that a restriction on the ability of ions to approach one another should also imply a restriction of ions to approach the surface of the particle. Taking this into account, the correction to the classical model becomes nonnegligible even for weakly charged particles suspended in low concentration electrolyte solutions [16, 17]. However, it is still necessary to consider effective ionic radii much larger than their hydrated values in order to fit experimental data.

The second was the use of more elaborate steric interaction models derived from hard sphere fluid theories, namely, the Carnahan-Starling equation [18] and its extension to the case of ion types with different sizes, namely, the "Boublik-Mansoori-Carnahan-Starling-Leland" (BMCSL) equation of state $[19,20]$.

Finally, it was realized that the existing theories actually do not imply that ions have a finite size: they only include restrictions on their ability to approach one another or the surface of the particle. On the contrary, a finite size ion should have a finite volume that can no longer be occupied by the suspending medium. The inclusion of this aspect led to the following main consequences:
(1) The presence of ions in the suspending medium should modify its permittivity. Moreover, since the ion concentration near a charged interface strongly depends on the distance to its surface, the electrolyte solution permittivity should also depend on this distance.

(2) A variable permittivity of the electrolyte solution leads to the appearance of a new force term acting on an ion since charges tend to move into regions of higher permittivity (a consequence of the so-called Born energy).

(3) The dielectric sphere representing an ion gets polarized by the local electric field and acquires a dipole moment. Therefore, an additional dielectrophoretic force acting on the dipole will appear wherever the local field is nonuniform.

The final result is an additional increase of the surface potential, which has a similar magnitude as that obtained taking only steric effects into account [21-26].

Inclusion of all the abovementioned effects is a formidable challenge from the computational point of view. Because of this, most of the existing studies consider the system behavior in equilibrium avoiding, furthermore, complications such as spherical symmetry.

Moreover, most studies only consider binary electrolyte solutions, in which the DEDL is basically composed of a single ionic species (the counterion) [26] so that the Bikerman and Carnahan-Starling expressions can be used to calculate ionion interactions. However, DEDLs in real systems are often composed of several ionic species. Under these conditions, the Bikerman and Carnahan-Starling expressions cease to be valid since they were deduced for single size spheres. Fortunately, the Carnahan-Starling expression has been extended to mixtures of spheres of unequal size, namely, the "Boublik-Mansoori-Carnahan-Starling-Leland" (BMCSL) equation of state $[19,20]$, making it possible to analyze the most general case. This theoretical model has been previously used by other authors but only in the case of binary electrolytes [27] or for mixed electrolytes assuming point co-ions [28]. Moreover, all the permittivity-related effects are neglected in these studies. The case of mixed electrolytes with finite counterion and co-ion sizes including permittivity-related effects is only considered in [29], to the best of our knowledge. However, this work uses an approximate procedure based on a generalization of the van der Waals equation to treat the steric interactions rather than the BMCSL equation. Moreover, the solution permittivity is calculated by means of a linear expression, and only the dielectrophoretic force is considered, while the Born force is neglected.

The aim of the present work is to analyze the structure of the DEDL for mixed electrolytes using a theoretical model that includes all the effects commented above. We show that the ionic size differences have a strong bearing on the diffuse part of the electric double layer structure. Larger counterions are expelled from the inner part of the diffuse double layer, while the concentration of the smaller ones increases. This leads to much lower surface charge values than predicted by 
Carnahan-Starling, bringing the theoretical results closer to those experimentally observed.

\section{Theory}

Let us consider an infinite uniformly charged plane interface immersed in an infinite solution made of $m$ ionic species represented by insulating spheres with hydrated radii $R_{i}$, effective permittivity $\varepsilon_{i}$, signed valences $z_{i}$, and bulk molar concentrations $c_{i}^{\infty}$ (in $\mathrm{mM}$ ), suspended in a continuous medium (water) with permittivity $\varepsilon_{w}$. The equilibrium DEDL is characterized by the local electric potential $\Psi$ and the ionic concentration $c_{i}$ profiles. Furthermore, due to the volume occupied by the ions, the electrolyte solution permittivity $\varepsilon_{e}$ also varies with the distance to the interface. This dependence can be estimated using the Maxwell mixture formula [30] which is quite accurate over the whole concentration range when the dispersions have a lower permittivity value than the suspending medium:

$$
\frac{\varepsilon_{e}-\varepsilon_{w}}{\varepsilon_{e}+2 \varepsilon_{w}}=\frac{4 \pi}{3} N_{A} \sum_{i=1}^{m} \frac{\varepsilon_{i}-\varepsilon_{w}}{\varepsilon_{i}+2 \varepsilon_{w}} R_{i}^{3} c_{i}
$$

where $N_{A}$ is the Avogadro number.

The electric potential is determined by the Poisson equation, written taking into account the spatial dependence of the electrolyte solution permittivity:

$$
\nabla \cdot\left(\varepsilon_{e} \vec{E}\right)=-\varepsilon_{e} \nabla^{2} \Psi-\nabla \Psi \cdot \nabla \varepsilon_{e}=e N_{A} \sum_{i=1}^{m} z_{i} c_{i},
$$

where $e$ is the elementary charge and $\vec{E}$ is the electric field.

On the other hand, the following competing macroscopic average forces (per mol) acting upon the ions determine the ionic concentrations:

(i) The electric force

$$
\vec{F}_{i}^{E}=-z_{i} e N_{A} \nabla \Psi
$$

(ii) The thermal force due to the random ionic movement

$$
\vec{F}_{i}^{T}=-k T N_{A} \nabla \ln \left(c_{i}\right),
$$

where $k$ is the Boltzmann constant and $T$ is the absolute temperature.

(iii) The steric force limiting the ability of ions to approach one another, which appears when ion size effects are taken into account

$$
\vec{F}_{i}^{S}=-k T N_{A} \nabla \mu_{i}^{e x},
$$

where $\mu_{i}^{e x}$ is the excess electrochemical potential.

(iv) The Born force that appears when the permittivity of the solution is allowed to change [31]: ions tend to move to regions of higher permittivity in order to lower their electrostatic energy

$$
\vec{F}_{i}^{B}=-N_{A} \nabla \frac{z_{i}^{2} e^{2}}{8 \pi \varepsilon_{e} R_{i}}=-\frac{z_{i}^{2} e^{2} N_{A}}{8 \pi R_{i}} \nabla\left(\frac{1}{\varepsilon_{e}}\right) .
$$

(v) The dielectrophoretic force that appears when ions are assumed to behave as dielectric spheres that become polarized by an external field acquiring an induced dipole moment $\vec{m}_{i}$ [32]

$$
\vec{F}_{i}^{D}=\left(\vec{m}_{i} \cdot \nabla\right) \vec{E}=2 \pi \varepsilon_{e} N_{A} R_{i}^{3} \frac{\varepsilon_{i}-\varepsilon_{e}}{\varepsilon_{i}+2 \varepsilon_{e}} \nabla\left(E^{2}\right) \text {. }
$$

In equilibrium, the sum of all the forces acting on each ion type must vanish, which leads to the following differential equations for plane geometry:

$$
\begin{aligned}
& \frac{d}{d x}\left[z_{i} \frac{e \Psi}{k T}+\ln \left(c_{i}\right)+\mu_{i}^{e x}+\frac{z_{i}^{2} e^{2}}{8 \pi k T R_{i} \varepsilon_{e}}\right] \\
& +\frac{2 \pi R_{i}^{3}}{k T} \varepsilon_{e} \frac{\varepsilon_{i}-\varepsilon_{e}}{\varepsilon_{i}+2 \varepsilon_{e}} \frac{d E^{2}}{d x}=0 .
\end{aligned}
$$

The solution of Equation (8) is

$$
c_{i}=K_{i} \exp \left(-\mu_{i}^{e x}\right) \exp \left(f_{i}\right) \exp \left(-\frac{z_{i} e \Psi}{k T}\right)
$$

where $K_{i}$ are integration constants,

$$
f_{i}=\frac{z_{i}^{2} e^{2}}{8 \pi k T R_{i}}\left(\frac{1}{\varepsilon_{e}^{\infty}}-\frac{1}{\varepsilon_{e}}\right)+\frac{4 \pi e R_{i}^{3}}{k T} \int_{x}^{\infty} \frac{\varepsilon_{e}\left(\varepsilon_{i}-\varepsilon_{e}\right)}{\varepsilon_{i}+2 \varepsilon_{e}} \frac{d \Psi}{d x} \frac{d^{2} \Psi}{d x^{2}} d x .
$$

$\varepsilon_{e}$ is given in Equation (1), and $\varepsilon_{e}^{\infty}=\varepsilon_{e}(x \longrightarrow \infty)$. Note that for $\varepsilon_{i}=\varepsilon_{w} \forall i \in\{1,2, \ldots, m\}$, all the effective ion permittivityrelated effects disappear.

These equations are solved considering as in [28] that the inner boundary of the DEDL is located at $x=0$ and using the usual boundary conditions:

Normal component of the electric field at $x=0$ :

$$
\left.\frac{d \Psi(x)}{d x}\right|_{x=0}=-\frac{\sigma_{s}}{\varepsilon_{e}(x=0)}
$$

where the electric field value is expressed in terms of a surface charge $\sigma_{S}$ by means of the Gauss law. Note that $\sigma_{S}$ does not represent the surface charge on the interface but rather minus the total charge of the DEDL.

Potential and ion concentration values far away from the interface:

$$
\begin{aligned}
& \Psi(x \longrightarrow \infty) \longrightarrow 0, \\
& c_{i}(x \longrightarrow \infty) \longrightarrow c_{i}^{\infty} .
\end{aligned}
$$

The last two conditions make it possible to determine the integration constants $K_{i}$ :

$$
K_{i}=c_{i}^{\infty} .
$$

In order to proceed, an analytical expression for the excess electrochemical potentials is required. The first theoretical model taking into account steric interactions among ions in the solution was proposed by Bikerman [14], who deduced the following expression for the excess electrochemical potential:

$$
\mu_{i}^{e x}=\mu^{e x}=\ln \left(\frac{1-\phi^{\infty}}{1-\phi}\right)
$$


where $\phi$ is the local value of the volume fraction occupied by all the ionic species in the solution:

$$
\phi=\frac{4 \pi N_{A}}{3} \sum_{i=1}^{m} R_{i}^{3} c_{i} .
$$

While the Bikerman expression is the first and simplest equation that allows to introduce steric interactions among ions into the theoretical model, later studies have produced more precise expressions. The most often used one is the Carnahan-Starling expression [18]:

$$
\mu_{i}^{e x}=\mu^{e x}=\frac{\phi\left(8-9 \phi+3 \phi^{2}\right)}{(1-\phi)^{3}}-\frac{\phi^{\infty}\left[8-9 \phi^{\infty}+3\left(\phi^{\infty}\right)^{2}\right]}{\left(1-\phi^{\infty}\right)^{3}} \text {. }
$$

While the Bikerman and Carnahan-Starling expressions for $\mu_{i}^{e x}$ are only valid when all the ionic species have the same size, the latter has been extended in 1970 by Boublik [19] and by Mansoori et al. [20] to the general case when different ionic species have different sizes. The obtained result can be written in the following form [28]:

$$
\begin{aligned}
\mu_{i}^{e x}+\mu_{i}^{\infty}= & -\left(1-12 R_{i}^{2} \frac{\xi_{2}^{2}}{\xi_{3}^{2}}+16 R_{i}^{3} \frac{\xi_{2}^{3}}{\xi_{3}^{3}}\right) \ln \left(1-\xi_{3}\right) \\
& +\frac{2 R_{i}\left(3 \xi_{2}+6 R_{i} \xi_{1}+4 R_{i}^{2} \xi_{0}\right)}{1-\xi_{3}} \\
& +\frac{12 R_{i}^{2} \xi_{2}\left(\xi_{2}+2 R_{i} \xi_{1} \xi_{3}\right)}{\xi_{3}\left(1-\xi_{3}\right)^{2}}-\frac{8 R_{i}^{3} \xi_{2}^{3}\left(\xi_{3}^{2}-5 \xi_{3}+2\right)}{\xi_{3}^{2}\left(1-\xi_{3}\right)^{3}},
\end{aligned}
$$

where

$$
\xi_{j}=\frac{2^{j-1} \pi N_{A}}{3} \sum_{i=1}^{m} c_{i} R_{i}^{j}, \quad j \in\{0,1,2,3\} .
$$

Note that $\xi_{3}$ denotes the total volume fraction occupied by ions in the solution ( $\phi$ in the Bikerman and Carnahan-Starling expressions (Equation (15)). On the other hand, Equation (17) reduces to the Bikerman expression for the ionic species $i$ if $R_{i}=0$ and at least one of the other species has a finite size and to the CarnahanStarling expression if it is assumed that all ionic species have the same hydrated radius.

\section{Results}

In what follows, we analyze the BMCSL theory predictions on the electric double layer structure and its differential capacitance (e.g., the variation of the surface charge density with the electric potential drop across the DEDL). We first consider binary electrolyte solutions with equal and different counterion and co-ion sizes and compare the obtained results with those obtained using the Carnahan-Starling theory. We then consider mixed electrolyte solutions with three ionic species.

The calculations were performed considering a bulk electrolyte concentration $c^{\infty}=10 \mathrm{mM}$ and a surface charge density $\sigma_{s}= \pm 0.4 \mathrm{C} / \mathrm{m}^{2}$. The values of the hydrated ionic radii were obtained from mobility measurements [33].

3.1. Binary Solutions. In order to compare the CarnahanStarling and BMCSL theory predictions, we first consider the simplest case of binary electrolyte solutions. We consider two situations: one with same size cations and anions $(\mathrm{KCl})$ and the other when these sizes are different $(\mathrm{NaCl})$. Figures 1 and 2 show the obtained results when all the permittivityrelated effects are taken into account. In these and in all the following figures, the words "full model" are used to express that both steric interactions and the permittivity-related effects are taken into account.

Figure 1 shows the counterion and co-ion concentration profiles in its upper and lower parts, both close to negative (left) and positive (right) interfaces. As can be seen, the counterion profiles predicted by the CS and BMCSL theories are undistinguishable from one another. This behavior is to be expected since, as shown in [26], the steric interactions in the DEDL depend almost exclusively on the counterion size in the case of binary electrolytes so that the system behaves as if both ions had the size of the counterion. Since under these conditions, the BMCSL expression reduces to that of $\mathrm{CS}$, it follows that both theories should lead to essentially the same results. However, a small difference does appear for coions in the $\mathrm{NaCl}$ system: the $\mathrm{BMCSL} \mathrm{Cl}^{-}$concentration is slightly higher at a negative interface, while the $\mathrm{Na}^{+}$is slightly lower at a positive one as compared to CS. This occurs because $\mathrm{Cl}^{-}$ions are smaller than $\mathrm{Na}^{+}$ions so that the steric repelling force is lower for $\mathrm{Cl}^{-}$and higher for $\mathrm{Na}^{+} \mathrm{co}-$ ions.

Figure 2 shows the electric potential profiles corresponding to the same cases as considered in Figure 1. As can be seen, the small co-ion concentration differences appearing in Figure 1 do not lead to any appreciable differences in the electric potential. This leads us to conclude that the predictions of the CS and the BMCSL theories are essentially the same in the case of binary electrolytes, as expected.

3.2. Mixed Electrolytes without Permittivity-Related Effects. We shall now compare the predictions of the CS and BMCSL theories in the case of solutions with three ionic species excluding at first all permittivity-related effects. This is achieved considering that $\varepsilon_{i}=\varepsilon_{w} \forall i \in\{1,2, \ldots, m\}$ so that $\varepsilon_{e}=\varepsilon_{w}$. We so consider a mixed electrolyte made of $\mathrm{NaCl}$ and $\mathrm{KCl}$ such that $c_{\mathrm{Cl}^{-}}^{\infty}=2 c_{\mathrm{Na}^{+}}^{\infty}=2 c_{\mathrm{K}^{+}}^{\infty}=10 \mathrm{mM}$.

Figure 3 shows the counterion and co-ion concentration profiles in its upper and lower parts, both close to negative (left) and positive (right) interfaces. As can be seen, the counterion profiles predicted by the CS and BMCSL theories are almost identical at the positive interface because the solution behavior depends almost exclusively on the size of the counterion, and there is only one: $\mathrm{Cl}^{-}$. As for the co-ions, both theories predict the same behavior for $\mathrm{K}^{+}$since its size is the same as that of the counterion. On the contrary, while CS predicts the same concentration for $\mathrm{Na}^{+}$as for $\mathrm{K}^{+}$, since steric interactions do 

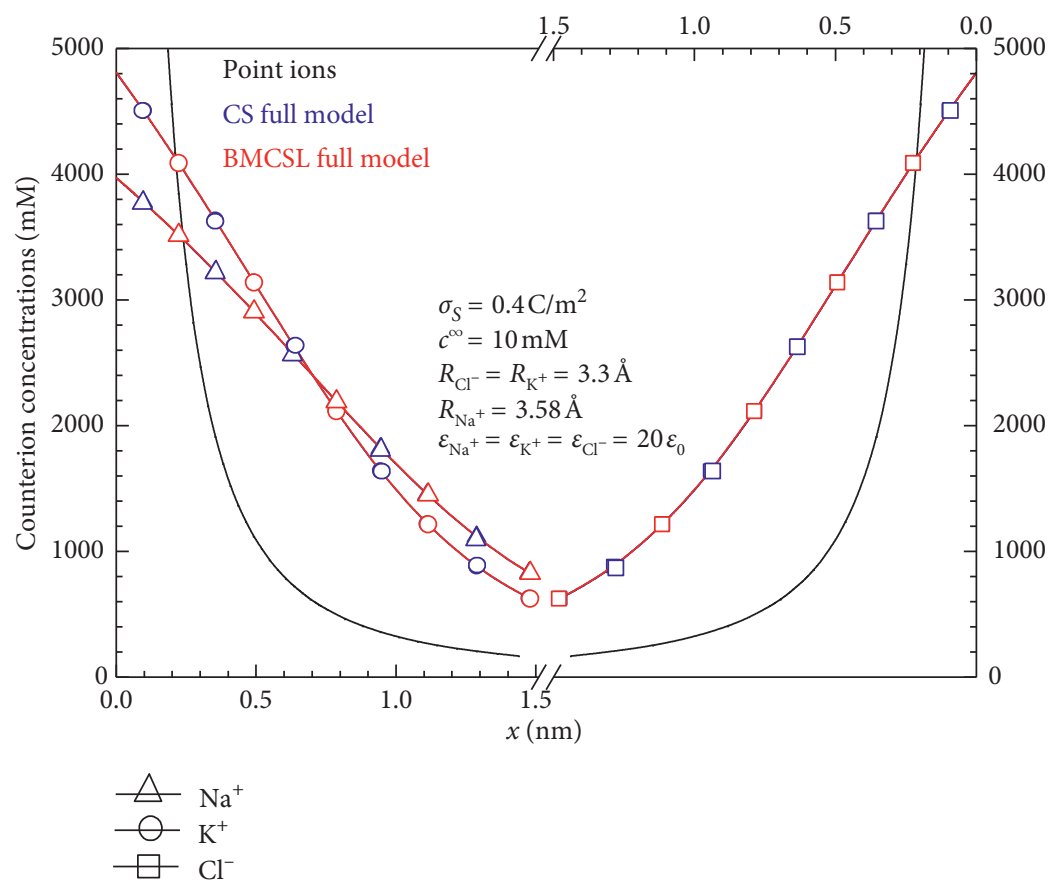

(a)

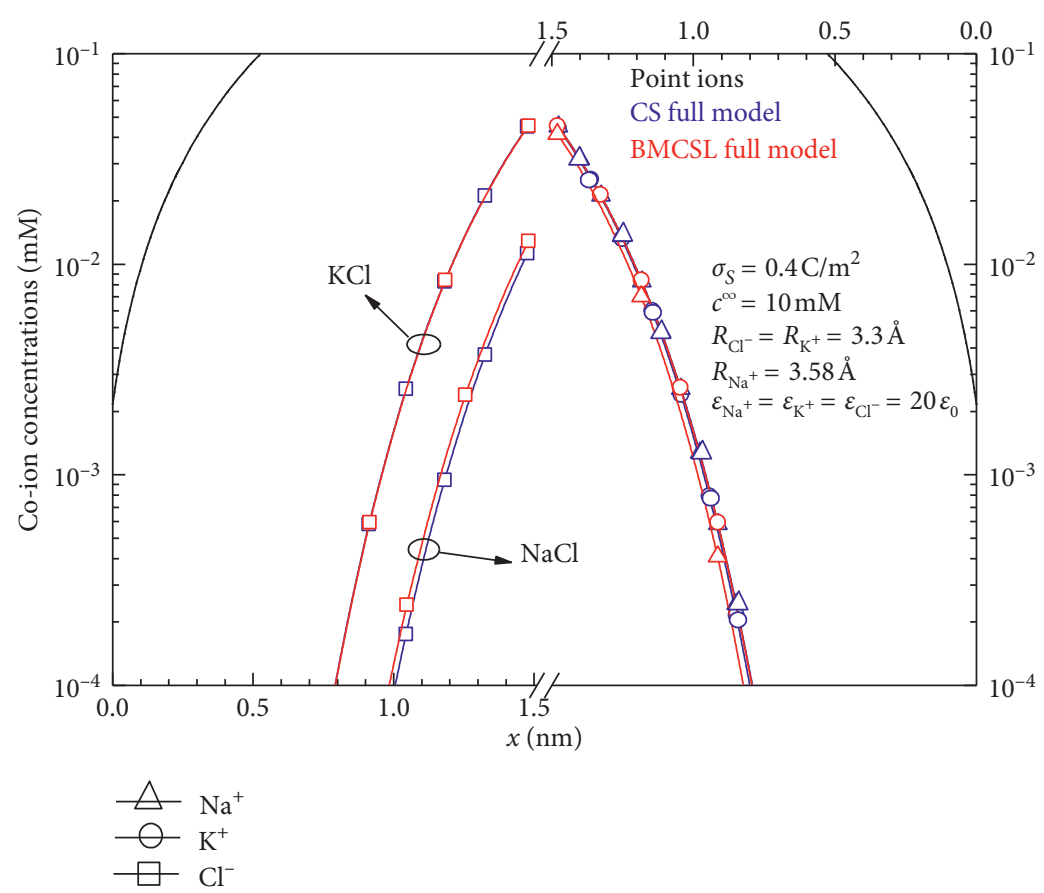

(b)

Figure 1: (a) Counterion and (b) co-ion concentration profiles in the diffuse electric double layers near negative (left) and positive (right) interfaces, calculated using the CS and BMCSL full models and aqueous $\mathrm{NaCl}$ and $\mathrm{KCl}$ solutions with the indicated parameters.

not depend on the individual ionic size, BMCSL predicts a lower $\mathrm{Na}^{+}$than $\mathrm{K}^{+}$concentration because its larger size implies greater repelling forces. A totally different situation arises at the negative interface where large differences appear between the counterion concentrations predicted by the two theories. The CS theory predicts equal $\mathrm{K}^{+}$and $\mathrm{Na}^{+}$ concentrations because the steric forces as well as the bulk concentrations are also the same. On the contrary, the
BMCSL theory predicts a much lower concentration of $\mathrm{Na}^{+}$ than $\mathrm{K}^{+}$near the interface due to the dependence of this model on the individual ionic size. While the $\mathrm{K}^{+}$concentration increases monotonously with decreasing distance to the interface, the $\mathrm{Na}^{+}$concentration reaches a maximum and starts to decrease due to its larger size. As for the co-ion $\left(\mathrm{Cl}^{-}\right)$concentration at the negative interface, it has a similar behavior as that in Figure 1: slightly higher 


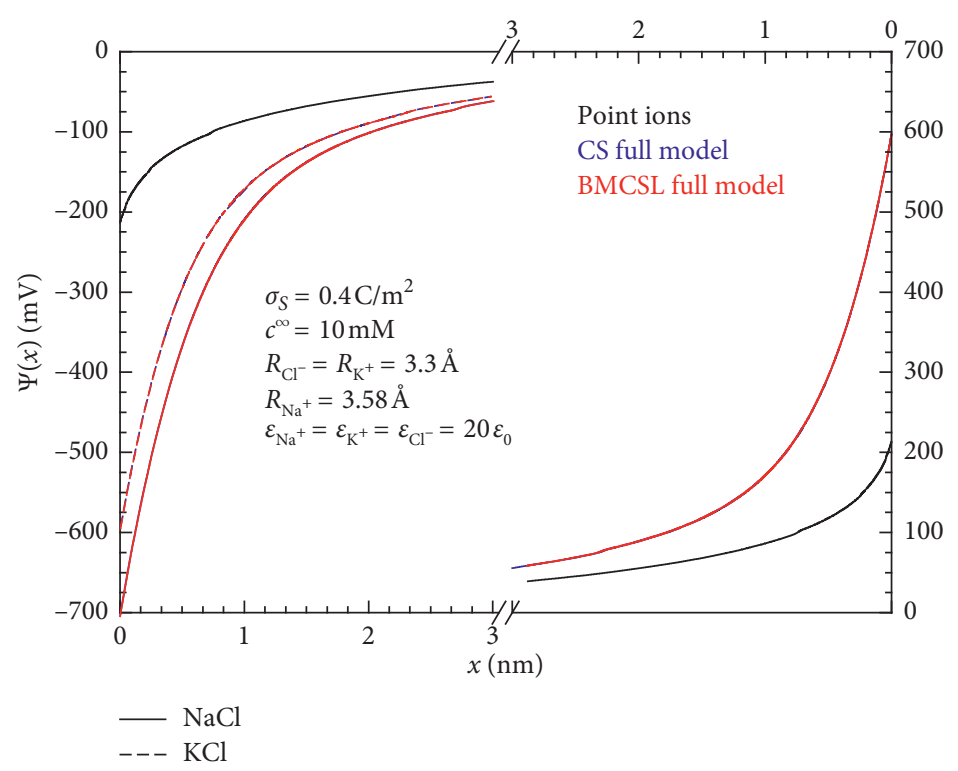

FIGURE 2: Electric potential profiles in the diffuse electric double layers near negative (left) and positive (right) interfaces, calculated using the CS and BMCSL full models and aqueous $\mathrm{NaCl}$ and $\mathrm{KCl}$ solutions with the indicated parameters.

according to the BMCSL than CS theories because of the presence of the larger $\mathrm{Na}^{+}$counterions.

Figure 4 shows the profiles corresponding to the total volume fraction of ions near negative and positive interfaces. As can be seen, according to both theories, these profiles coincide at the positive interface since, as already mentioned, the steric forces are determined almost exclusively by the counterion sizes and there is only one counterion: $\mathrm{Cl}^{-}$. As for the negative interface, both theories predict a higher volume fraction of ions than at a positive one, which occurs because the $\mathrm{Na}^{+}$ion is larger than both the $\mathrm{K}^{+}$and $\mathrm{Cl}^{-}$ions. Moreover, BMCSL predicts a lower volume fraction than CS because of the higher concentration of the smaller $\mathrm{K}^{+}$than the larger $\mathrm{Na}^{+}$ions (Figure 3).

Figure 5 shows the electric potential profiles for the same system as in Figures 3 and 4. As expected, no differences between the CS and BMCSL predictions can be seen at the positive interface because the counterion $\left(\mathrm{Cl}^{-}\right)$concentrations are almost identical (Figure 3). On the contrary, small differences appear at the negative interface: BMCSL predicts a slightly lower (in modulus) potential drop than CS because the double layer is thinner due to a higher relative concentration of the smaller ion $\left(\mathrm{K}^{+}\right)$(Figures 3 and 4 ).

We so conclude that for mixed electrolytes, the BMCSL model generally leads to a thinner electric double layer than CS because the steric forces are higher for the larger ions that tend to be expelled from the double layer so that it is mostly made of the smallest counterions. However, for the considered case when both electrolytes have the same bulk concentration and the surface charge density is moderate, the resulting potential differences are small. Therefore, the expected predictions of the two models regarding the differential capacitance should also be similar.

This situation changes, however, when we consider nonsymmetric bulk electrolyte concentrations and high potential values. This can be seen in Figure 6 that shows the potential drop across the DEDL, $V^{d}$, as a function of the surface charge density for two complementary bulk counterion concentrations: rich in $\mathrm{Na}^{+}$or rich in $\mathrm{K}^{+}$. The potential drop at the positive interface has been omitted almost entirely since both considered models lead to essentially the same results because the double layer is then made almost exclusively of $\mathrm{Cl}^{-}$ions, which have the same bulk concentration in both considered systems. For point ions, the same results are obtained for the two considered cases because both counterion species have the same valence and the total bulk counterion concentration is also the same. For the rich in $\mathrm{K}^{+}$case, the CS and BMCSL models also predict almost identical results since the double layer is mostly made of the smaller $\mathrm{K}^{+}$ions. The situation changes drastically for the rich in $\mathrm{Na}^{+}$case since CS predicts that the relative composition of both counterion species in the double layer remains the same as in the bulk, i.e.,

$$
\frac{c_{\mathrm{Na}^{+}}}{c_{\mathrm{K}^{+}}}=\frac{c_{\mathrm{Na}^{+}}^{\infty}}{c_{\mathrm{K}^{+}}^{\infty}} .
$$

This behavior is also true for the BMCSL model but only for small surface charge densities. For higher charge values, the larger $\mathrm{Na}^{+}$ions are progressively expelled from the double layer (Figure 3), while the $\mathrm{K}^{+}$concentration grows. This decreases the thickness of the DEDL, leading to a lower potential drop.

While this difference shown in Figure 6 appears to be small, it leads to important consequences for the differential capacitance of the diffuse double layer:

$$
C_{d}=\frac{d \sigma_{S}}{d V^{d}}
$$

This can be seen in Figure 7 that shows this magnitude as a function of the potential drop across the DEDL for the same cases as considered in Figure 6. For point ions, the same behavior is obtained for the two systems that is 

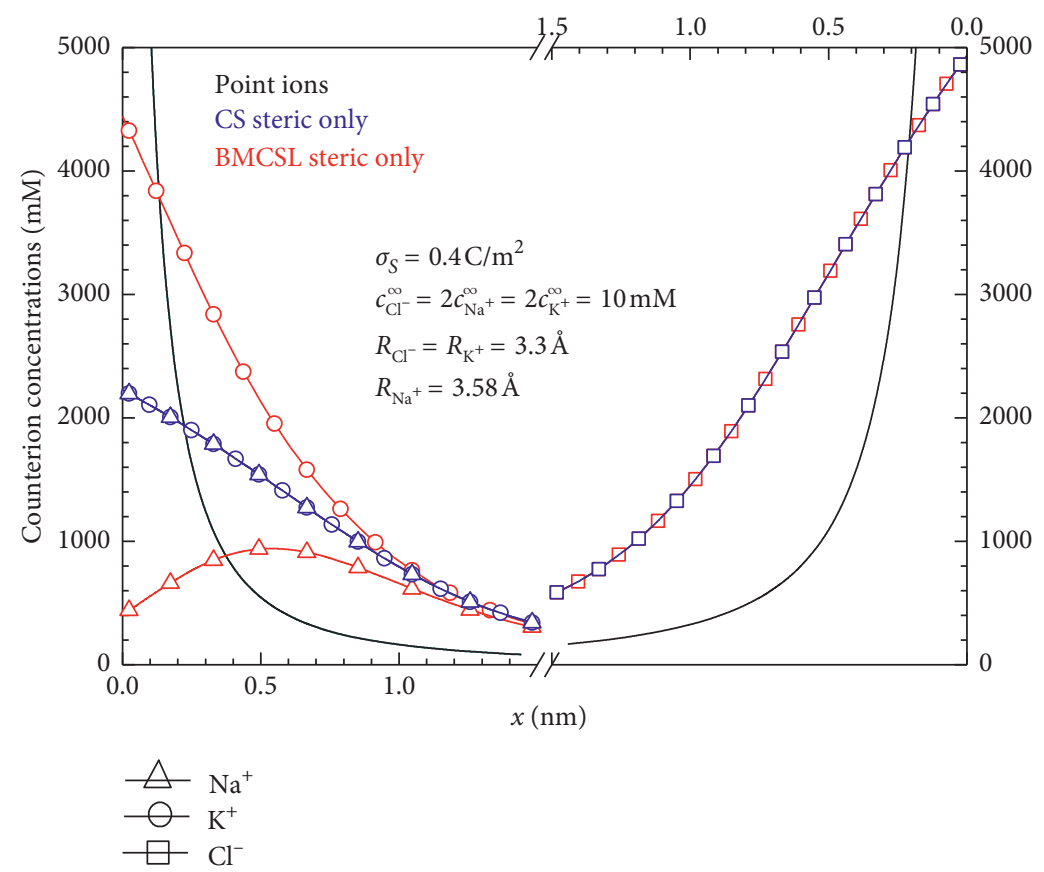

(a)

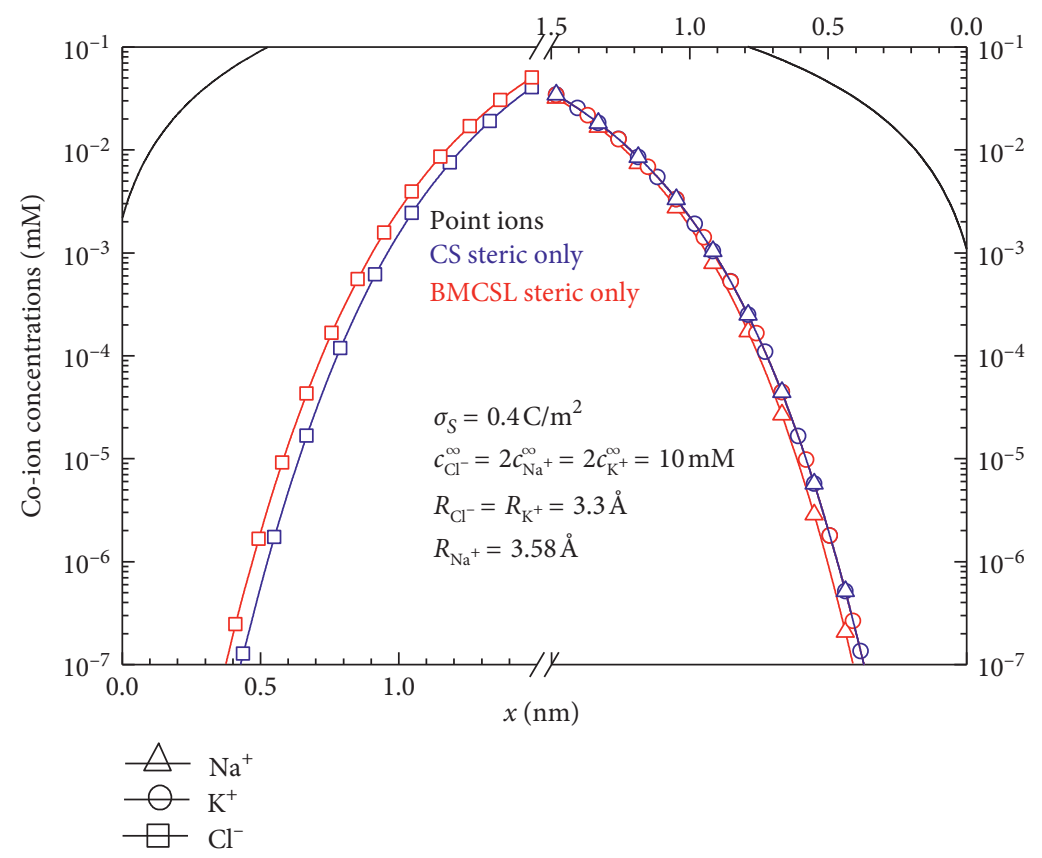

(b)

FIGURE 3: (a) Counterion and (b) co-ion concentration profiles in the diffuse electric double layers near negative (left) and positive (right) interfaces, calculated using the CS and BMCSL steric interaction only models and mixed aqueous $\mathrm{NaCl}$ and $\mathrm{KCl}$ solutions with the indicated parameters.

characterized by an unbounded growth of the differential capacitance with increasing absolute potential drop value. The CS model predicts a different behavior for the two considered cases that is mostly determined by the counterion with the highest bulk concentration. This is why the maximum value of the differential capacitance is larger and is attained at a higher (in modulus) potential for the rich in $\mathrm{K}^{+}$ system, due to its lower double layer thickness. Finally, the
BMCSL model leads to results that are very similar to CS but only for the rich in $\mathrm{K}^{+}$system since, according to both models, the double layer is then mainly composed of $\mathrm{K}^{+}$ions. On the contrary, for the rich in $\mathrm{Na}^{+}$system, both models lead to similar results for low (in modulus) potentials only. For increasing potentials, the larger $\mathrm{Na}^{+}$ ion is progressively expelled from the diffuse double layer, which decreases its thickness increasing the differential 


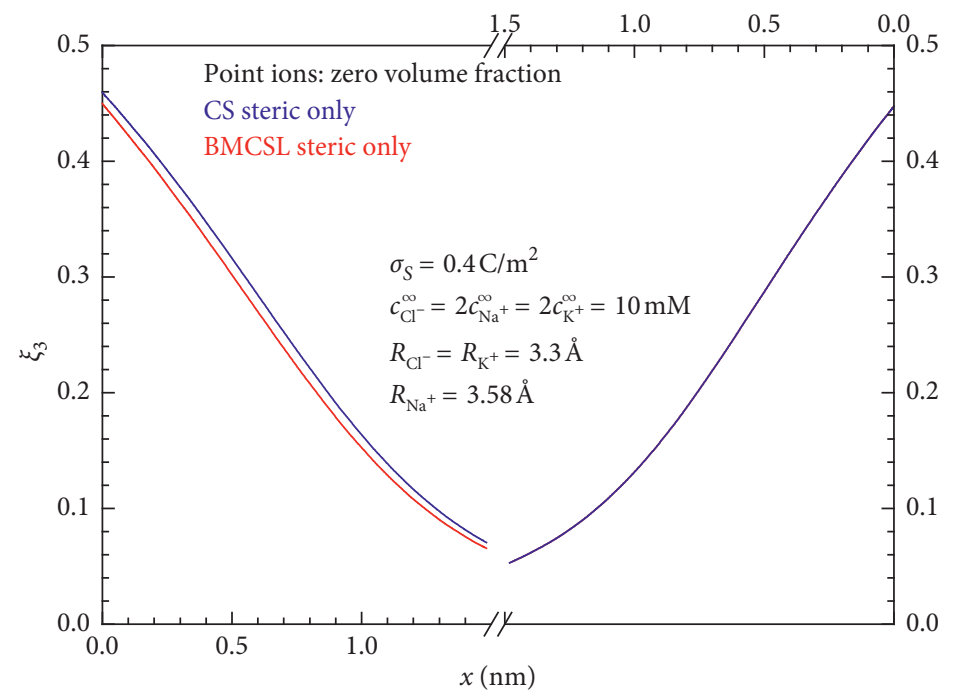

Figure 4: Total ionic volume fraction profiles in the diffuse electric double layers near negative (left) and positive (right) interfaces, calculated using the CS and BMCSL steric interaction only models and mixed aqueous $\mathrm{NaCl}$ and $\mathrm{KCl}$ solutions with the indicated parameters.

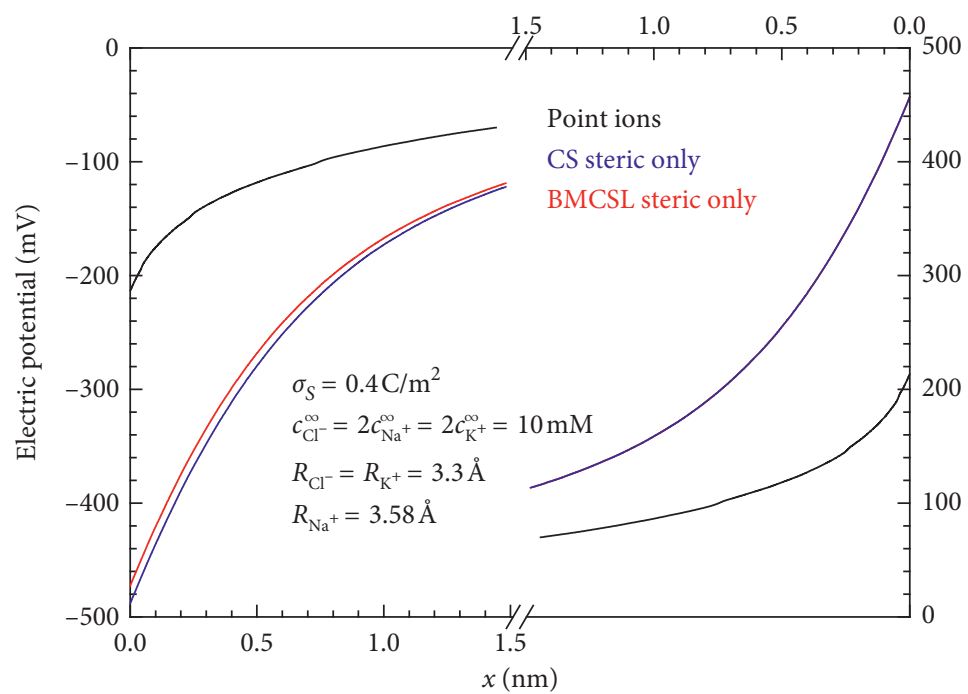

FIGURE 5: Electric potential profiles in the diffuse electric double layers near negative (left) and positive (right) interfaces, calculated using the CS and BMCSL steric interaction only models and mixed aqueous $\mathrm{NaCl}$ and $\mathrm{KCl}$ solutions with the indicated parameters.

capacitance. Note that for very high (in modulus) potentials, BMCSL actually predicts higher differential capacitance values than CS since, under these conditions, the relative $\mathrm{Na}^{+}$ concentration in the double layer drops below the constant value predicted by CS (Equation (19)).

\subsection{Mixed Electrolytes including Permittivity-Related Effects.} We shall now extend the comparison of the predictions of the BMCSL and CS theories considering that ions behave as dielectric spheres with permittivity values different from that of water. We so assume that for all the ionic species, $\varepsilon_{i} / \varepsilon_{0}=20$. We shall first analyze the case of a mixed electrolyte with equal compositions of $\mathrm{NaCl}$ and $\mathrm{KCl}$, just as in Figures 3-5.
Figure 8 shows the counterion concentration profiles at negative and positive interfaces predicted by the considered theories. This figure is similar to Figure 3, except for the absence of the co-ion concentrations that have been omitted because they are relatively unimportant for the double layer behavior as already discussed. The permittivity-related effects do not alter the qualitative behavior predicted by the BMCSL model. The dielectrophoretic force tends to diminish the concentration of all the ionic species close to the interface. This is why the $\mathrm{Na}^{+}$ concentration for $x=0$ has a lower value than in Figure 3. As for the concentration behavior of $\mathrm{K}^{+}$that actually increases for $x=0$, the interpretation is more complex: the dielectrophoretic force tends to lower the $\mathrm{K}^{+}$concentration but the decrement of the $\mathrm{Na}^{+}$concentration decreases the 


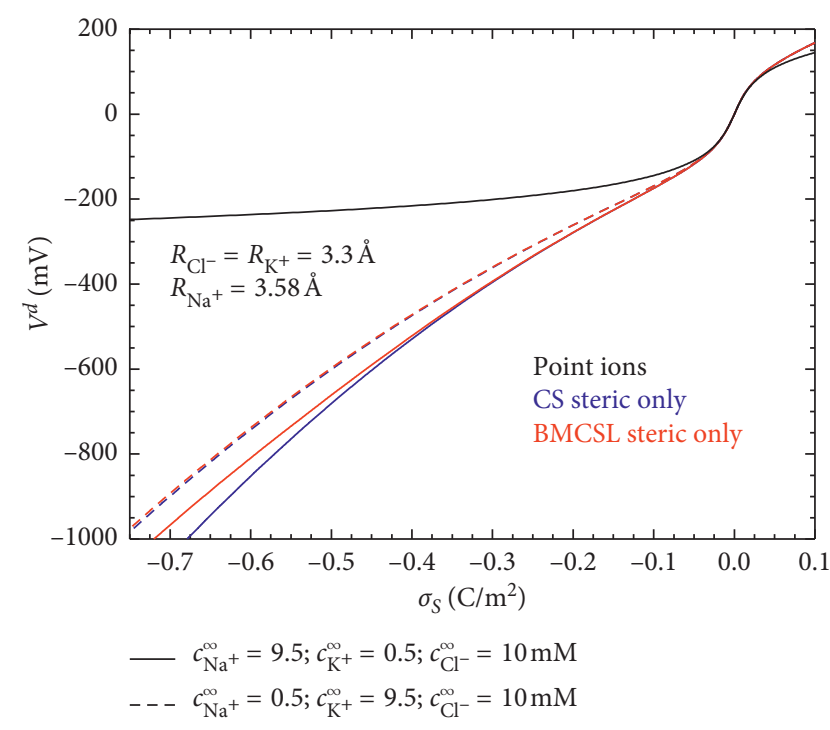

Figure 6: Dependence of the electric potential drop across the diffuse electric double layer on the surface charge density calculated using the CS and BMCSL steric interaction only models and mixed aqueous $\mathrm{NaCl}$ and $\mathrm{KCl}$ solutions with the indicated parameters.

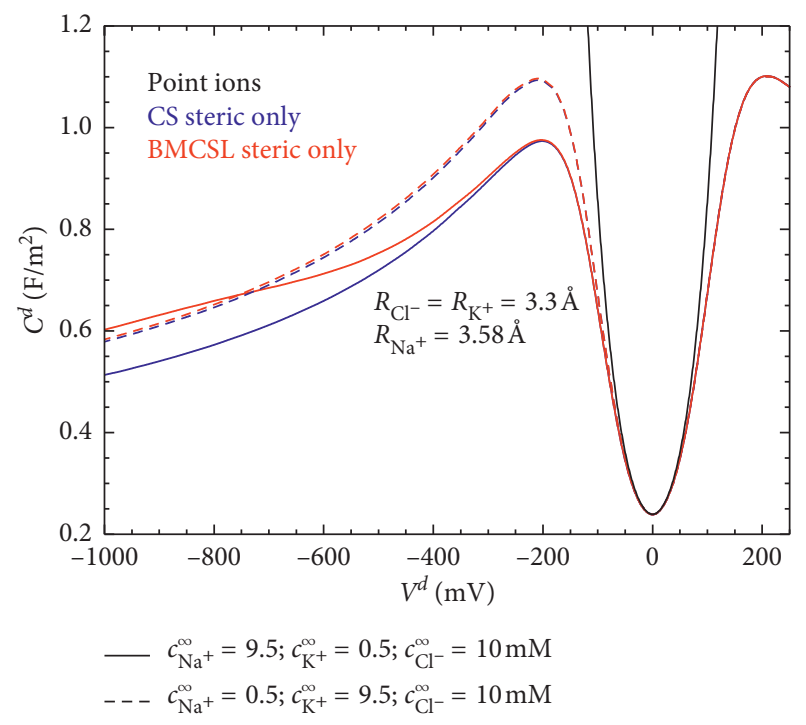

FIGURE 7: Dependence of the differential capacitance of the diffuse electric double layer on the potential drop across this layer, calculated using the CS and BMCSL steric interaction only models and mixed aqueous $\mathrm{NaCl}$ and $\mathrm{KCl}$ solutions with the indicated parameters.

steric repelling force acting on the $\mathrm{K}^{+}$ions tending to increase their concentration. In the considered case, the second phenomenon is stronger than the first so that the $\mathrm{K}^{+}$ concentration close to the interface ultimately increases. On the other hand, permittivity-related effects have a much stronger bearing on the CS predictions that change qualitatively: the $\mathrm{Na}^{+}$concentration attains a maximum and diminishes close to the interface. This happens because of the size dependence of the dielectrophoretic force that is proportional to the cube of the ionic radius. Because of this,

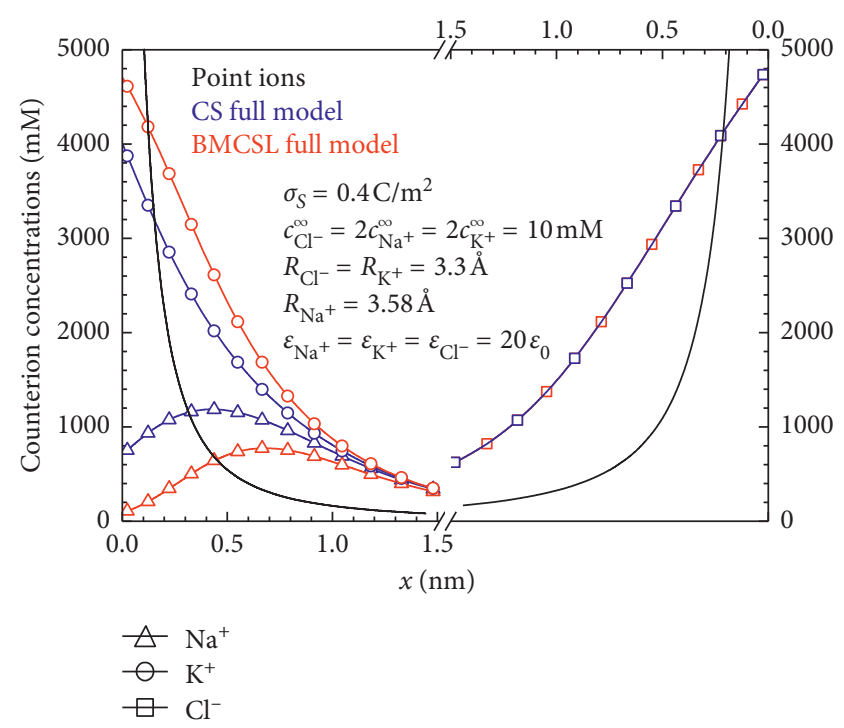

FIgURE 8: Counterion concentration profiles in the diffuse electric double layers near negative (left) and positive (right) interfaces, calculated for the CS and BMCSL full models and mixed aqueous $\mathrm{NaCl}$ and $\mathrm{KCl}$ solutions with the indicated parameters.

even when steric interactions are independent of the counterion size according to CS, the repelling dielectrophoretic force is stronger for $\mathrm{Na}^{+}$than for $\mathrm{K}^{+}$, leading to a concentration behavior that is qualitatively similar to that predicted by BMCSL.

Because of this behavior, both models also lead to similar potential drop versus surface charge density relationships. This can be seen in Figure 9, which is identical to Figure 6 except for the inclusion of all the permittivity-related effects. For both the BMCSL and CS theories, the use of the dielectric sphere model strongly increases the potential drop across the diffuse double layer: the $-1000 \mathrm{mV}$ value is attained for 0.5 rather than $0.7 \mathrm{C} / \mathrm{m}^{2}$ (Figure 6). This is due to two reasons: the increase of the double layer thickness mainly due to the dielectrophoretic force and the decrease of the double layer permittivity.

The differences between the CS and BMCSL theories become apparent in Figure 10, where the differential capacitance is represented as a function of the potential drop for the same systems as in Figure 9. The increment of the double layer thickness and the decrement of its permittivity lead in both cases to an overall decrease of the differential capacitance, clearly seen when comparing Figures 10 and 7 . However, for high (in modulus) values of the diffuse layer voltage, the CS results for the $\mathrm{Na}^{+}$rich system tend now to those corresponding to the system rich in $\mathrm{K}^{+}$because for both systems, the dielectrophoretic force finally expels the largest counterion from the double layer.

In conclusion, the dielectric sphere model leads to the following modifications of the CS and BMCSL theories:

(1) The effects due to different ionic sizes already present in the BMCSL theory are incremented since both the steric and the dielectrophoretic forces increase with the ionic size. Because of this, the increment of the 


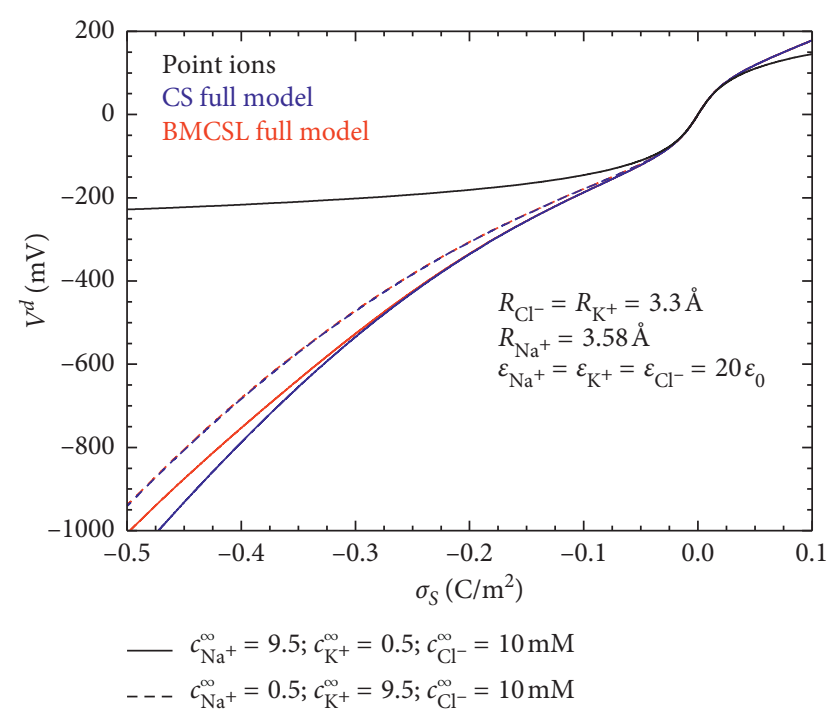

Figure 9: Dependence of the electric potential drop across the diffuse electric double layer on the surface charge density calculated using the $\mathrm{CS}$ and BMCSL full models and mixed aqueous $\mathrm{NaCl}$ and $\mathrm{KCl}$ solutions with the indicated parameters.

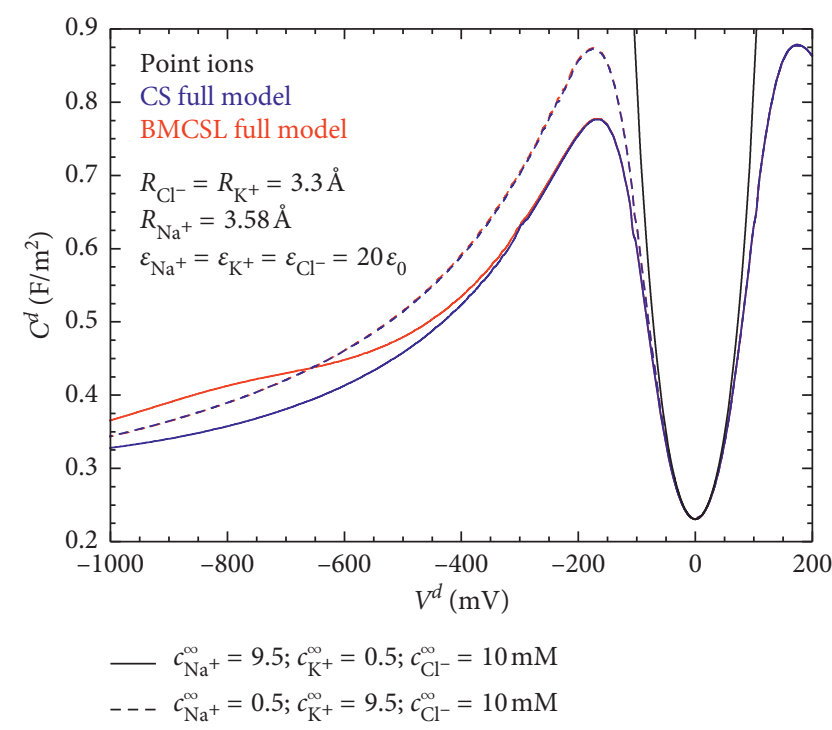

Figure 10: Dependence of the differential capacitance of the diffuse electric double layer on the potential drop across this layer calculated using the CS and BMCSL full models and mixed aqueous $\mathrm{NaCl}$ and $\mathrm{KCl}$ solutions with the indicated parameters.

potential drop across the diffuse double layer and the corresponding decrement of the differential capacitance occur at lower surface charge densities.

(2) The effects due to different ionic sizes that are absent in the CS theory appear when it is combined with the dielectric sphere model. Because of this, the qualitative behavior of the CS theory becomes similar to that of BMCSL. However, the quantitative results remain quite different except for very low or very large surface charge values.

\section{Conclusions}

In this work, we present a theoretical account based on the Poisson-Boltzmann equation of the influence of the finite ionic size on the diffuse double layer properties of binary and mixed electrolyte solutions. This is done combining for the first time the "Boublik-Mansoori-Carnahan-Starling-Leland" (BMCSL) theory for the steric interactions among ions with all the effects that result from the representation of ions as dielectric spheres. These include the dependence of the solution permittivity on the local ionic concentration, calculated here by means of the Maxwell mixture formula, and the appearance of two additional forces acting on the ions, namely, the Born and the dielectrophoretic forces that depend on the permittivity and the electric field gradients, respectively.

We compare the predictions of the BMCSL and the often used Carnahan-Starling (CS) steric interaction theories. The obtained results show that in the case of binary electrolyte solutions, there are practically no differences between the results obtained using either of these two theories, both combined with all the permittivity-related effects.

In the case of mixed electrolytes, and without considering the permittivity-related effects, the CS concentration profiles for both counterion species only differ by a constant factor: the ratio of the bulk concentrations of the two counterion species. On the contrary, according to the BMCSL theory, for sufficiently high surface charges and irrespective of the relative bulk concentrations, the smaller counterion concentration always surpasses the concentration of the larger one.

When the CS theory is combined with all the permittivity-related effects, a dependence on the individual ionic sizes appears mainly due to the repelling dielectrophoretic force that is proportional to the ionic volume. This leads to a qualitative behavior that is similar to that predicted by the BMCSL theory without the permittivityrelated effects. However, the dependence on the ionic sizes of the full BMCSL theory that includes the permittivityrelated effects is much stronger so that the larger counterions are expelled from the inner part of the diffuse double layer at much lower surface charges than for CS.

\section{Data Availability}

The data used to support the findings of this study are available from the corresponding author upon request.

\section{Conflicts of Interest}

The authors declare that there are no conflicts of interest regarding the publication of this paper.

\section{References}

[1] D. Andelman, "Electrostatic properties of membranes: the Poisson-Boltzmann theory, chapter 12," in Handbook of Biological Physics, R. Lipowsky and E. Sackmann, Eds., vol. 1, Elsevier Science, Amsterdam, Netherlands, 1995. 
[2] J. Lyklema, Fundamentals of Colloid and Interface Science. Solid/Liquid Interfaces, Academic Press, Vol. II, Academic Press, London, UK, 1995.

[3] M. V. Fedorov and A. A. Kornyshev, "Ionic liquids at electrified interfaces," Chemical Reviews, vol. 114, no. 5, pp. 2978-3036, 2014.

[4] H. Helmholtz, "Ueber einige Gesetze der Vertheilung elektrischer Ströme in körperlichen Leitern mit Anwendung auf die thierisch-elektrischen Versuche (in German)," Annalen der Physik und Chemie, vol. 165, no. 6, pp. 211-233, 1853.

[5] G. Gouy, "Sur la constitution de la charge électrique à la surface d'un électrolyte," Journal de Physique Théorique et Appliquée, vol. 9, no. 1, pp. 457-468, 1910.

[6] D. L. Chapman, "A contribution to the theory of electrocapillarity," The London, Edinburgh, and Dublin Philosophical Magazine and Journal of Science, vol. 25, no. 148, pp. 475-481, 1913.

[7] O. Stern, "Zur Theorie der electrolytischen Doppelschicht," Zeitschrift für Elektrochemie, vol. 30, p. 508, 1924.

[8] D. C. Grahame, "The electrical double layer and the theory of electrocapillarity," Chemical Reviews, vol. 41, no. 3, pp. 441501, 1947.

[9] M. A. V. Devanathan, "A theory of the electrical double layer and the interpretation of differential capacity curves," Transactions of the Faraday Society, vol. 50, p. 373, 1954.

[10] J. OM. Bockris, M. A. V. Devanthan, and K. Mueller, "On the structure of charged interfaces," Proceedings of the Royal Society A: Mathematical, Physical and Engineering Sciences, vol. 274, no. 1356, pp. 55-79, 1963.

[11] B. E. Conway, "Transition from "supercapacitor" to "battery" behavior in electrochemical energy storage," Journal of The Electrochemical Society, vol. 138, no. 6, pp. 1539-1548, 1991.

[12] G. Valette, "Double layer on silver single crystal electrodes in contact with electrolytes having anions which present a slight specific adsorption part 1 the (110) face," Journal of Electroanalytical Chemistry and Interfacial Electrochemistry, vol. 122, pp. 285-297, 1981.

[13] M. Z. Bazant, M. S. Kilic, B. D. Storey, and A. Ajdari, "Towards an understanding of induced-charge electrokinitics at large applied voltages in concentrated solutions," Advances in Colloid and Interface Science, vol. 152, no. 2, pp. 48-88, 2009.

[14] J. J. Bikerman, "Structure and capacity of electrical double layer,” Philosophical Magazine, vol. 33, p. 384, 1942.

[15] A. S. Khair and T. M. Squires, "Ion steric effects on electrophoresis of a colloidal particle," Journal of Fluid Mechanics, vol. 640 , p. 343, 2009.

[16] J. J. López-García, M. J. Aranda-Rascón, C. Grosse, and J. Horno, "Equilibrium electric double layer of charged spherical colloidal particles: effect of different distances of minimum ion approach to the particle surface," Journal of Physical Chemistry B, vol. 114, no. 22, pp. 7548-7556, 2010.

[17] J. J. López-García, M. J. Aranda-Rascón, C. Grosse, and J. Horno, "Electrokinetics of charged spherical colloidal particles taking into account the effect of ion size constraints," Journal of Colloid and Interface Science, vol. 356, no. 1, pp. 325-330, 2011.

[18] N. F. Carnahan and K. E. Starling, "Equation of state for nonattracting rigid spheres," Journal of Chemical Physics, vol. 51, no. 2, pp. 635-636, 1969.

[19] T. Boublik, "Hard-sphere equation of state," Journal of Chemical Physics, vol. 53, no. 1, pp. 471-472, 1970.

[20] G. A. Mansoori, N. F. Carnahan, K. E. Starling, and T. W. Leland, "Equilibrium thermodynamic properties of the mixture of hard spheres," Journal of Chemical Physics, vol. 54, no. 4, pp. 1523-1525, 1971.

[21] J. J. López-García, C. Grosse, and J. Horno, "The PoissonBoltzmann description of the electrical double layer including ion size effects," Langmuir, vol. 27, no. 23, pp. 13970-13974, 2011.

[22] M. M. Hatlo, R. Van Roij, and L. Lue, "The electric double layer at high surface potentials: the influence of excess ion polarizability," EPL, vol. 97, no. 2, p. 28010, 2012.

[23] J. J. López-García, C. Grosse, and J. Horno, "Influence of the dielectrophoretic force in mixed electrical double layers," Journal of Colloid and Interface Science, vol. 405, pp. 336-343, 2013.

[24] E. Gongadze and A. Iglic, "Asymmetric size of ions and orientational ordering of water dipoles in electric double layer model-an analytical mean-field approach," Electrochimica Acta, vol. 178, pp. 541-545, 2015.

[25] Y. Nakayama and D. Andelman, "Differential capacitance of the electric double layer: the interplay between ion finite size and dielectric decrement," Journal of Chemical Physics, vol. 142, no. 4, article 044706, 2015.

[26] J. J. López-García, J. Horno, and C. Grosse, "Differential capacitance of the diffuse double layer at electrode-electrolyte interfaces considering ions as dielectric spheres: part I. Binary electrolyte solutions," Journal of Colloid and Interface Science, vol. 469, pp. 531-539, 2017.

[27] L. Lue, N. Zoeller, and D. Blankschtein, "Incorporation of nonelectrostatic interactions in the Poisson-Boltzmann equation," Langmuir, vol. 15, no. 11, pp. 3726-3730, 1999.

[28] P. M. Biesheuvel and M. Soestbergen, "Counterion volume effects in mixed electrical double layers," Journal of Colloid and Interface Science, vol. 316, no. 2, pp. 490-499, 2007.

[29] G. Minton and L. Lue, "The influence of excluded volume and excess ion polarizability on the capacitance of the electric double layer," Molecular Physics, vol. 114, no. 16-17, pp. 2477-2491, 2016.

[30] J. C. Maxwell, A Treatise on Electricity and Magnetism, Clarendon, Vol. 1, Clarendon, Oxford, UK, 1892.

[31] M. Born, "Volumen und Hydratationswerme der Ionen," Zeitschrift für Physik, vol. 1, no. 1, pp. 45-48, 1920.

[32] H. A. Pohl, "Some effects of nonuniform fields on dielectrics," Journal of Applied Physics, vol. 29, pp. 1182-1188, 1958.

[33] E. R. Nightingale, "Phenomenological theory of ion solvation. Effective radii of hydrated ions," Journal of Physical Chemistry, vol. 63, pp. 1381-1387, 1959. 


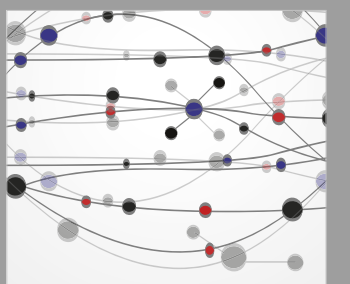

The Scientific World Journal
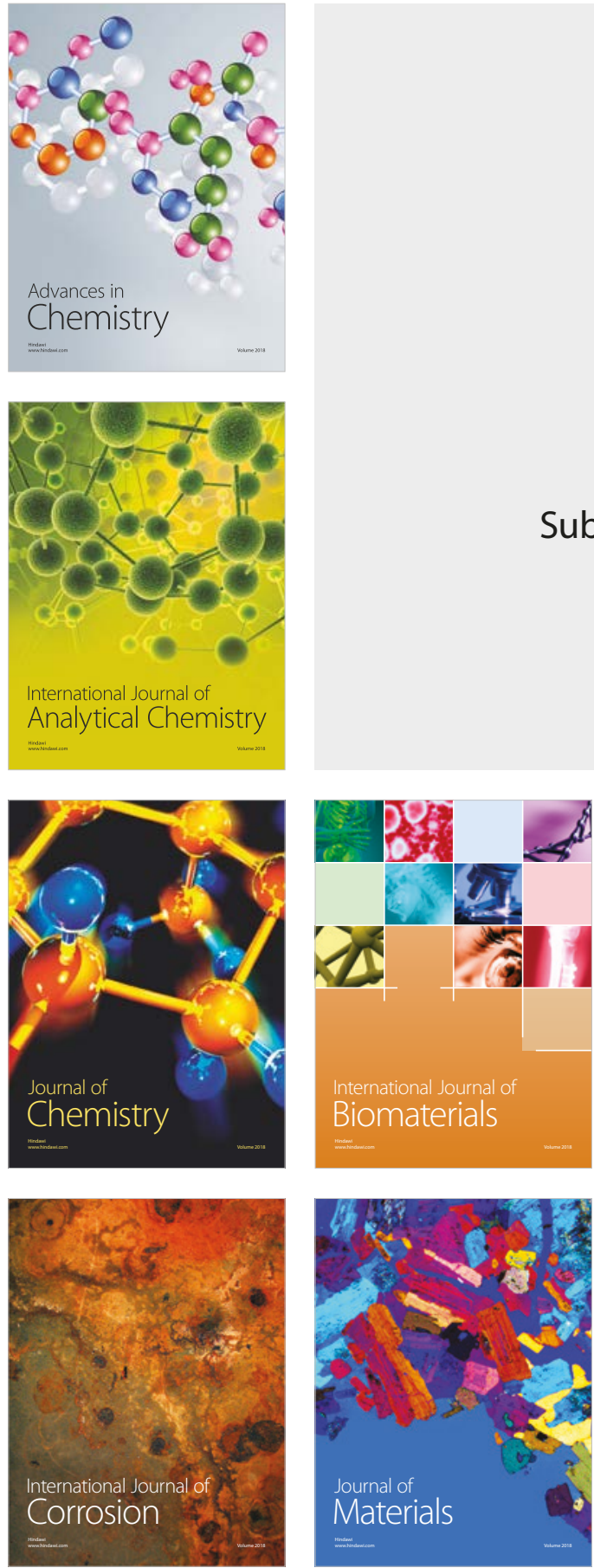

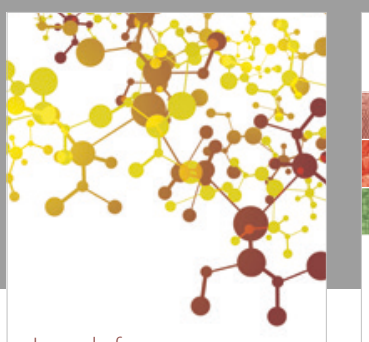

Journal of

Applied Chemistry
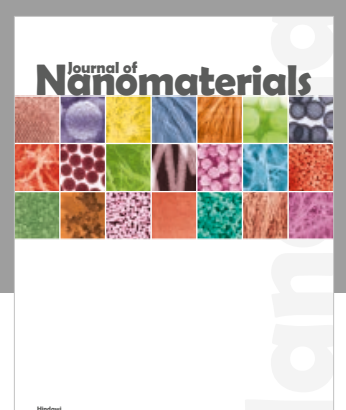

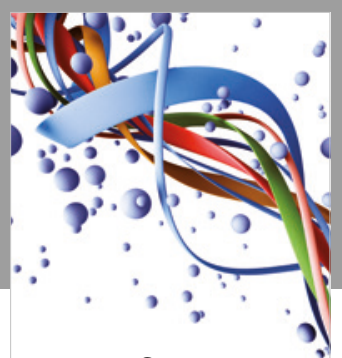

Scientifica

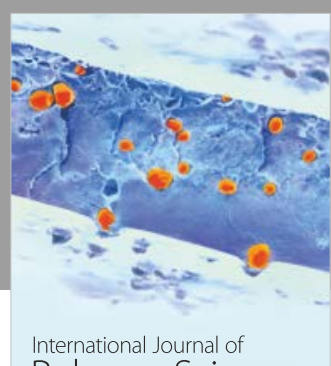

Polymer Science

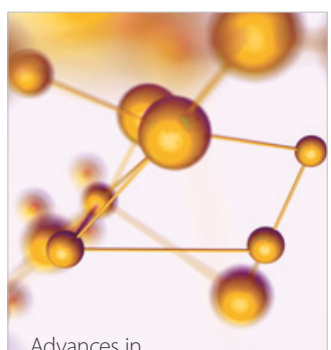

Physical Chemistry
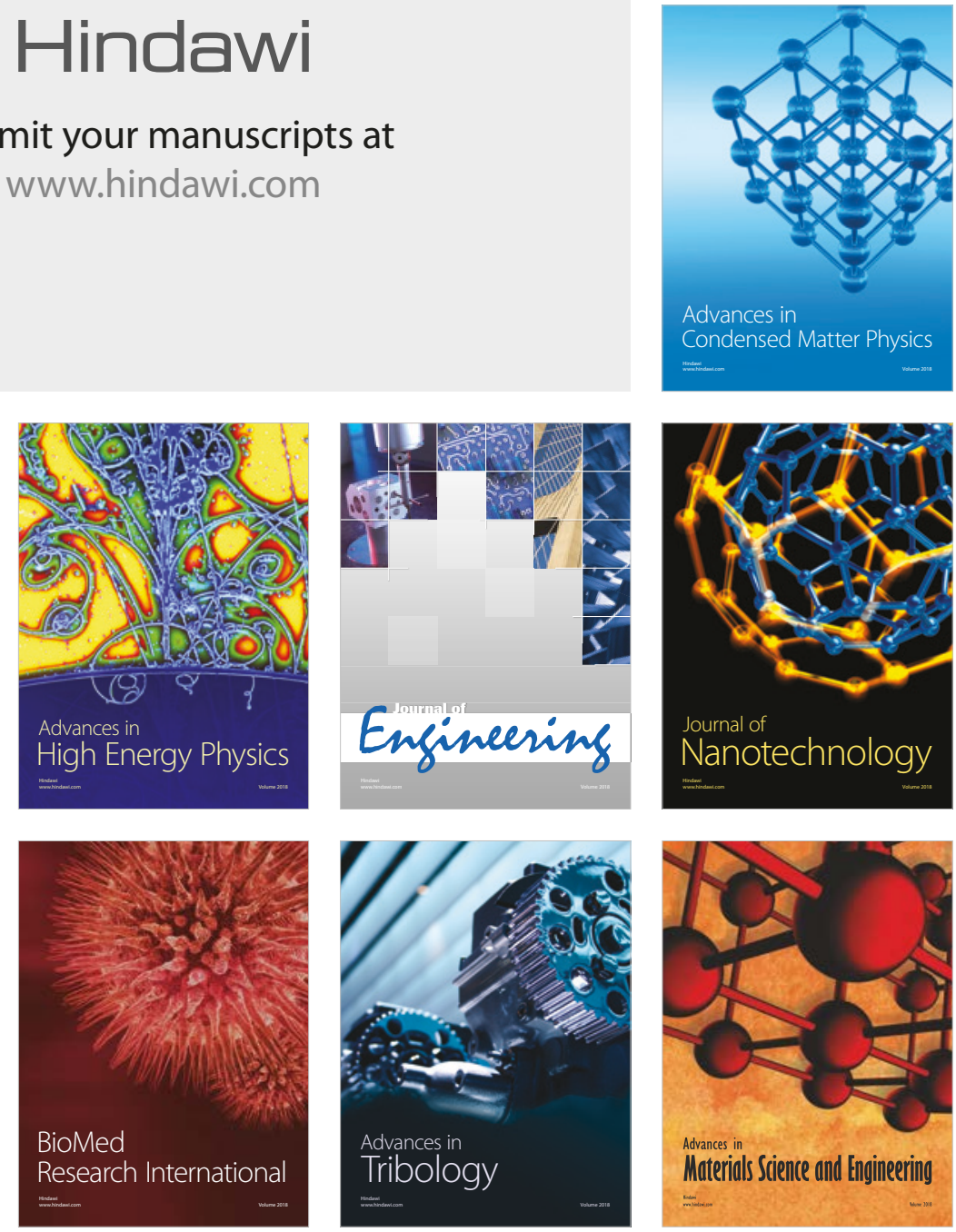